\title{
The Effect of Clocking on Compressor Noise Generation
}

\author{
Kypros Milidonis*, Bernhard Semlitsch $†$ and Tom Hynes ${ }^{\ddagger}$ \\ Whittle Laboratory, Department of Engineering, University of Cambridge \\ 1 JJ Thomson Avenue, Cambridge, CB3 ODY, United Kingdom
}

The effect of stator clocking on the acoustic noise generation characteristics in an axial high-pressure compressor is analysed. A realistic geometry with one-and-a-half stages is assessed using high fidelity and low-order numerical methods for different clocking positions at approach operating conditions. The compressor efficiency and the acoustic noise emission is found to vary insignificantly between the simulated clocking configurations. Nonetheless, the pressure distribution is altered significantly right upstream of the inlet guide vanes. Although the cut-on modes exhibit at least $10 \mathrm{~dB}$ higher amplitudes, the cut-off modes contribute decisively to the wave pattern in the near field. Optimal acoustic liner design can expand on the differently evolving interference pattern of acoustic waves at discrete frequencies. The low-order model is found to predict the directionality of the acoustic waves and the cut-on criteria for the individual modes in excellent agreement with the high fidelity simulations. However, the phase cannot be estimated due to the simplicity of the low-order formulation.

\section{Introduction}

$\prod$ HE demand for high thrust on future turbofan engines implies high rotational shaft speeds. The resultant high flow velocities give rise to noise sources at the compressor blade tips with tonal characteristics. This discrete frequency noise is considered to be particularly annoying. ${ }^{1,2}$ Consequently, reduction of tonal compressor noise is required, especially at near ground operating conditions, i.e. during take-off and approach. This calls for novel design approaches to further reduce acoustic noise pollution.

Rotor and stator interaction triggers tonal noise, where two sources are predominant; ${ }^{3}$ (viscous) wake interception and (inviscid) potential flow unsteadiness. The latter is caused by the blockage of blades, which affects the pressure field. Acoustic waves are emitted with mutual reaction of the rotating blade pressure distribution. As indicated in Fig. 1, the wakes of the upstream blades give rise to a fluctuating downwash at the impeding blade. Hence,

\footnotetext{
${ }^{*} \mathrm{PhD}$, Research Associate.

${ }^{\dagger} \mathrm{PhD}$, Research Associate.

${ }^{\ddagger} \mathrm{PhD}$, Reader.
} 


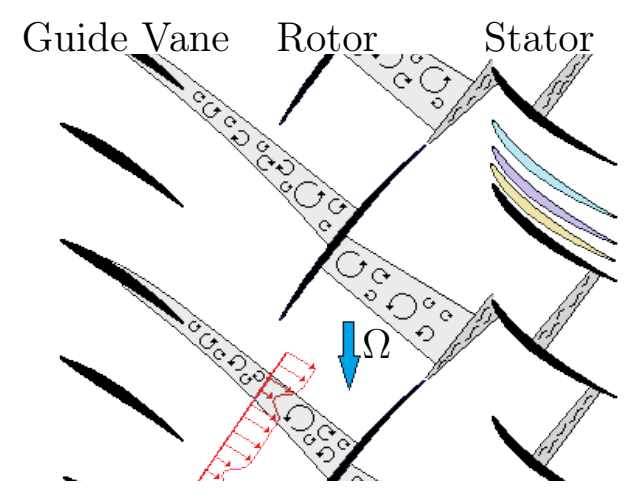

Figure 1. Stage geometry and stator clocking configurations (indicated in colour code) along with typical instantaneous wake dispersion pattern.

the blade loading is unsteady and generates acoustic noise. The comparative importance of these two mechanisms is governed by the gap extent between blade rows. ${ }^{4}$

These two acoustic wave generation mechanisms couple to tonal modes related to multiples of the Blade Passing Frequency (BPF). With dissimilar numbers of stator and rotor blades, the disturbance interaction and hence the noise generation exhibits a certain phase shift causing circumferentially distorted modes. Tyler and Sofrin ${ }^{5}$ derived a formulation to predict the excited circumferential modes, $m_{\theta}$, on a single stage; $m_{\theta}=n N_{R}+\nu N_{S}$, where $N_{R}$ and $N_{s}$ are the counts of rotor and stator blades, respectively. The order of harmonic, $n$, and $\nu$ are integers. Low mode numbers, with small circumferential wave distortion, dominate the tonal noise in the far field. Contrary, high mode numbers are likely to be cut-off and do not radiate into the far field. Hence, optimal blade counts can be determined for acoustic noise reduction using this strategy.

These circumferential modes can be affected in a multistage setup. Copeland et al. ${ }^{6}$ showed experimentally that adding a downstream stator to a stator/rotor configuration can result in lower sound pressure levels. With multistage compressors, the mode amplitudes may partially cancel out when their phase relationship is favourably inline with modes from other stages. The phase relation of modes can be altered by clocking or indexing, i.e. circumferentially revolving, the downstream stator with respect to the upstream stator, as shown in Fig. 1. The idea of clocking for acoustic noise reduction is dated back to 1972, when Walker and Oliver ${ }^{7}$ experimentally revealed that two sets of wakes can mutually cancel the greater part of the velocity defect at certain points, and thus reduce noise. Later, Schmidt and Okiishi ${ }^{8}$ reported that the level of discrete frequency noise in the area upstream of the first stator could be varied appreciably by the relative circumferential positioning of stationary vanes. More recently, Kovalev et al. ${ }^{9}$ presented experimental results of a 1.5 stage compressor, testifying that clocking may be used to attenuate tonal noise. For a transonic axial multi-stage fan, Kamiyoshi and $\mathrm{Kaji}^{10}$ demonstrated analytically that acoustic waves can cancel partially out reproducing their earlier experimental clocking observations, ${ }^{11}$ where noise reduction up to $16.8 d B$ could be achieved.

The effect of clocking on the axial compressor performance has been investigated by many research groups. Among the first were Barankiewicz and Hathaway ${ }^{12}$ studying experimentally a low-pressure 4.5-stage axial compressor. They showed insignificant impact of clocking on the overall efficiency (less than $0.2 \%$ at both, peak efficiency and peak pressure operating conditions), which was rather attributed to measurements and experimental setup uncertain- 
ties. That the relation of clocking position and efficiency has a sinusoidal shape with typical variations within the range of 0.2 up to $2.7 \%,{ }^{13-15}$ is nowadays commonly accepted. ${ }^{16-18}$ However, controversial findings have been reported regarding the optimal impingement location of the upstream stator wake on the downstream stator vane to maximise efficiency. For example, Gundy-Burlet et al. ${ }^{19}$ and Dorney et al. ${ }^{17}$ concluded that the optimal combination of high efficiency occurred when the upstream stator wake was convected along the pressure surface of the downstream stator. On the contrary, He et al., ${ }^{20}$ Hongyan et al., ${ }^{16}$ Key et al., ${ }^{18}$ and Fruth et al. ${ }^{21}$ observed maximum efficiency when the first stage/rotor wakes impinge on the leading edges of the second-stage rotor/stator, respectively. Städing et al. ${ }^{15}$ found best performance when the upstream rotor or stator wakes propagate near mid-passage of the clocked blade row. Fruth et al. ${ }^{21}$ highlight the fact that the wake impingement location is not a trivial criteria, as wake impingement on a twisted blade might occur at different relative locations over the span.

Summarising, clocking has been found to be an effective noise reduction technique in axial compressors, while observations were predominantly empirical. Numerically inexpensive approaches are required to predict the near field sound pressure levels for novel acoustic liner and low-noise compressor design. Within the present work, we investigate the impact of stator clocking on the acoustic near field on the Variable Inlet Guide Vane (VIGV) casing. Therefore, high fidelity calculations are employed to parametrically simulate the unsteady flow in a 1.5-stage compressor for different clocking configurations. The circumferential mode amplitudes are compared for the blade passing frequency and its first two harmonics showing the consequence for the far field noise. The clocking sensitivity on the wave pattern in the near field is revealed using Fourier decomposition, which is of interest for the application of acoustic liners. Finally, low-order modelling is utilised to predict these sound pressure maps revealing promising qualities as a future design tool.

\section{Methodology}

In this section, the numerical approaches, i.e. high fidelity and low-order methods, are described. The geometry under investigation consists of a realistic one-and-a-half stage compressor (i.e. VIGV/rotor/stator) with engine representative blade profiles, axial blade row gaps, and a typical S-duct upstream of the VIGV's. The number of VIGV's and downstream stators are identical $\left(N_{V I G V}=N_{S}=40\right)$, whereas the rotor blade count is $\left(N_{R}=32\right)$. These numbers, with a common denominator of blade counts, allow a domain reduction to $1 / 8^{\text {th }}$ of the full annulus configuration (i.e. 5 VIGVs, 4 rotors, and 5 stators).

\section{II.A. High-fidelity flow simulations}

The strategy used to predict the VIGV/rotor/stator interaction tone noise is based on high fidelity, time-accurate simulation of the Navier-Stokes equations using the Rolls-Royce code HYDRA. ${ }^{22}$ The main advantage of such approaches is their ability to handle multi-frequency sources in one single simulation, while taking into account non-linear phenomena. The calculations are carried out in the relative reference frame of each blade row, and sliding planes are employed at the $\mathrm{VIGV} /$ rotor and the rotor/stator interfaces.

The dual time-stepping technique was used for time marching. A single rotor passing period, based on $1 / 8^{\text {th }}$ of the annulus geometry, has been resolved with 1920 time-steps 
(i.e. 60 per rotor passage). This is sufficient to distinguish frequencies corresponding to $\mathrm{VIGV} /$ rotor and rotor/stator interaction in the relative frame of reference. Each outer timestep demanded on average 4 to 6 inner iterations to reach a drop in the residual by three orders of magnitude. The simulation was run for 5 to 6 complete periods on the Cambridge University High Performance Computing Cluster using 512 cores. The Spalart-Allmaras turbulence model was employed in all calculations.

The flow field was initialised with converged solutions of single blade passage steady-state Reynolds-Averaged Navier Stokes (RANS) calculations. For the steady-state simulations, the interfaces between the blade rows were treated as mixing planes.

After converging the unsteady simulation to the desired operating point with sliding planes and the $1 / 8^{\text {th }}$ of the full annulus domain, the Fourier coefficients for the blade passing frequency and its first two harmonics have been extracted. These are computed internally using the full temporal and spatial resolution of the calculation over one additional full revolution run. The sound pressure is then extracted to quantify the acoustic noise level.

\section{II.A.1. Case setup, numerical mesh, quality criteria, operating and boundary conditions}

The computational domain is outlined in Fig. 2. Several stator clocking positions have been simulated. However, only three clocking position cases are shown here, $0 \%, 25 \%$, and $50 \%$ as well as a fourth case in which the downstream stator is not present. All the other cases follow the presented trends. (Zero percent clocking refers to the scenario when the wake of the VIGVs impinges the leading edges of the downstream stators.) A parallel annulus section was attached upstream of the S-duct. In addition, the stator domain was extruded downstream as shown in Fig. 2. These two parallel-annulus sections were added to the computational domain to incorporate two mesh regions: I) a fine grid region able to resolve frequencies up to the second harmonic of the blade passing frequency at the rotor's specific speed and II) a buffer-sponge zone region designed to minimise any artificial numerical reflections originating from the inlet and exit boundary. To maintain the correct inflow characteristics, the hub and casing walls of the inlet buffer-sponge zone are set to be inviscid.

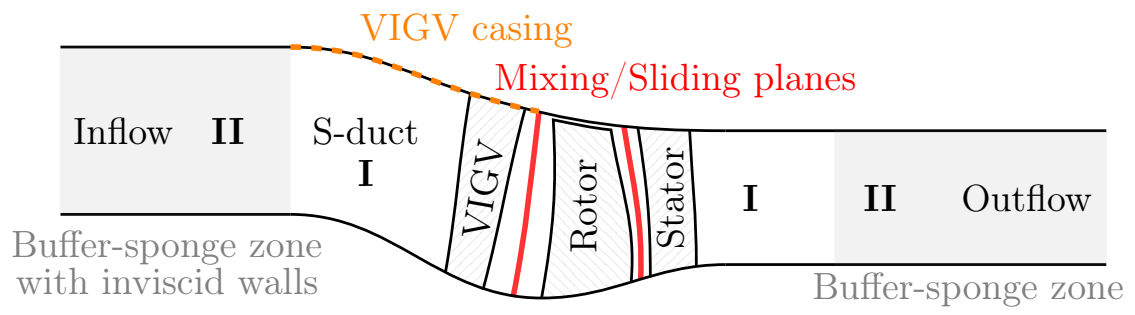

Figure 2. Meridional profile sketch of the computational domain indicating the fine (I) and buffer-sponge (II) mesh regions.

The inlet boundary is treated as non-reflecting subsonic inflow, while the exit boundary is handled as a non-reflecting radial equilibrium outflow. The required exit flow function at the rotor exit is achieved by adjusting the exit static pressure profile, which sets the required pressure ratio $\left(p_{\text {out }} / p_{\text {in }}\right)$. All cases were converged to the same operating point, which is a typical high-power approach certification condition. ${ }^{23}$ The inflow profiles correspond to the discharge angles of the swirl vanes. The rotor speed is $92.16 \%$ of the corrected design speed.

The VIGV/rotor interaction noise can include frequencies up to three times the blade passing frequency. This results in short acoustic wavelengths, which require sufficient nodal 
density to be resolved. The grid quality was ensured by allocating 20 to 30 grid points per theoretical acoustic wavelength, where the acoustic wavelengths were calculated as presented in Section II.B. The main mesh criterion for the span-wise direction was to resolve the boundary layer adequately, since the radial component of the acoustic mode tends to have larger wavelengths. Hence, fewer nodes would be necessary only satisfying the recommended number of grid points per acoustic wavelength.

All computational domains were meshed using the Rolls-Royce mesh generation tool, PADRAM. ${ }^{24}$ A block-structured grid topology was used, i.e. an $\mathrm{H}-\mathrm{O}-\mathrm{H}$ topology with a body-fitted O-mesh around the rotor blades and stator vanes to achieve $y^{+}$values less than unity. Adequate span-wise mesh layers are added within the tip gap (whose size is $0.11 \%$ of the span) using the butterfly-mesh technique to enhance the local quality of the mesh. The mesh was conformally matched at the VIGV/rotor and rotor/stator interfaces (i.e. mixing and sliding plane). The computational domain was discretised with approximately 9 million cells for a single VIGV passage, 5.4 million cells for a single rotor passage and 8 million cells for a single stator passage. Hence, approximately 106.7 million cells are required for the $1 / 8^{\text {th }}$ of the full annulus configuration.

\section{II.B. Low-order representation}

To extract the acoustic wave directionality of individual modes from the three-dimensional flow solution is a challenge. ${ }^{25,26}$ Likewise, low-order models can be utilised to predict the individual directivity of the circumferential modes emitted by the rotor/stator interaction. Such formulations can be obtained from the linearised Navier-Stokes equations ${ }^{\mathrm{a}}$, which are valid for small perturbation amplitudes. With the energy, mass, and momentum conservation equations, the dispersion relation for acoustic pressure waves, $p^{\prime}$, can be derived in the cylindrical coordinate system, i.e. $x, r, \theta$,

$$
\frac{\partial^{2} p^{\prime}}{\partial x^{2}}+\frac{1}{r^{2}} \frac{\partial^{2} p^{\prime}}{\partial \theta^{2}}=\frac{p^{\prime}}{a^{2}}\left(\frac{\partial}{\partial t}+v_{x} \frac{\partial}{\partial x}+\frac{v_{\theta}}{r} \frac{\partial}{\partial \theta}\right)^{2}
$$

where $v_{x}$ and $v_{\theta}$ are the axial and azimuthal velocities respectively, and $a$ is the local speed of sound. Substituting solutions of the form $p^{\prime}=f(x) e^{i \omega t+i m_{\theta} \theta}$ into Eq. 1 gives,

$$
\left(1-\left(\frac{v_{x}}{a}\right)^{2}\right) \frac{d^{2} f(x)}{d x^{2}}-2 i \frac{v_{x}}{a}\left(\frac{\omega r}{a}+m_{\theta} \frac{v_{\theta}}{a}\right) \frac{d f(x)}{d x}+\left(\left(\frac{\omega r}{a}+m_{\theta} \frac{v_{\theta}}{a}\right)^{2}-m_{\theta}^{2}\right) f(x)=0 .
$$

The angular frequency, $\omega$, is $m_{\theta} N_{R} \Omega$ and $m_{\theta}$ is the circumferential harmonic of distortion. The two roots of this equation are the axial wavenumbers, $k_{x(1,2)}$,

$$
k_{x(1,2)}=\frac{\frac{v_{x}}{a}\left(\frac{\omega}{a}+\frac{m_{\theta}}{r} \frac{v_{\theta}}{a}\right)}{1-\left(\frac{v_{x}}{a}\right)^{2}} \pm \frac{i \sqrt{\left(\left(\frac{m_{\theta}}{r}\right)^{2}\left(1-\left(\frac{v_{x}}{a}\right)^{2}\right)-\left(\frac{\omega}{a}+\frac{m_{\theta}}{r} \frac{v_{\theta}}{a}\right)^{2}\right)}}{1-\left(\frac{v_{x}}{a}\right)^{2}}
$$

representing solutions of the form $e^{i k_{x} x}$. If $\left(\frac{\omega r}{a}+m_{\theta} \frac{v_{\theta}}{a}\right)^{2} \geq m_{\theta}^{2}\left(1-\left(\frac{v_{x}}{a}\right)^{2}\right)$, the axial wavenumbers remain solely real valued and are thus cut-on. Axial wavenumbers with imaginary components are cut-off and consequently not propagating. With the solution of the

\footnotetext{
${ }^{a}$ Hence, the flow variables are decomposed into a mean and fluctuation components, where the fluctuations are labelled by a prime.
} 
form, $e^{i \omega t+i k_{x(1,2)} x+i m_{\theta} \theta}$, the inclination angle of the waves can be calculated by $\tan (\alpha)=$ $\frac{k_{x(1,2)} r}{m_{\theta}}$. Further, the dispersion relation, Eq. 1, can be rewritten as,

$$
\left(\frac{\omega r}{a}+r k_{x(1,2)} \frac{v_{x}}{a}+m_{\theta} \frac{v_{\theta}}{a}\right)^{2}=r^{2} k_{x(1,2)}^{2}+m_{\theta}^{2} .
$$

Thereby, the inclination angle of all upstream propagating waves can be predicted. However, their amplitudes need to be extracted from the high fidelity simulations, if sound pressure level maps are desired. Noteworthy is that this estimation does not consider the phase relation between modes.

\section{Results}

Clocking affects both principal acoustic noise generation mechanisms of blade row interaction. Firstly, the wake of the upstream stators convects through the downstream stator vanes at different locations. This can be observed in terms of entropy contours in Fig. 3. Amplified entropy contours can be noted, which impinge on the downstream stator leading edge for the $0 \%$ clocked configuration and evolves approximately at mid-passage for the $50 \%$ clocked configuration. Cyclic flow separation, due to alteration of flow incidence angles by the upstream stator wake, on the downstream stator cannot be observed. Also the impact on the wake width of the downstream stator is marginal. Noteworthy is that the entropy contours exhibit negligible variations. Thus, the compressor performance differs insignificantly (less than $0.02 \%$ ) between the investigated configurations.

Secondly, via their blockage, blades generate a potential pressure field, which interacts the neighbouring pressure field due to the spinning of the rotor. Thereby, acoustic noise is generated. Figure 4 shows that the hydrodynamic pressures remain at similar overall amplitudes for the different clocking configurations. Nonetheless, significant blade-to-blade loading variations can be observed at the downstream stator, which occur phase shifted due to clocking. Such alterations reproduce at the blade tip and influence the potential pressure
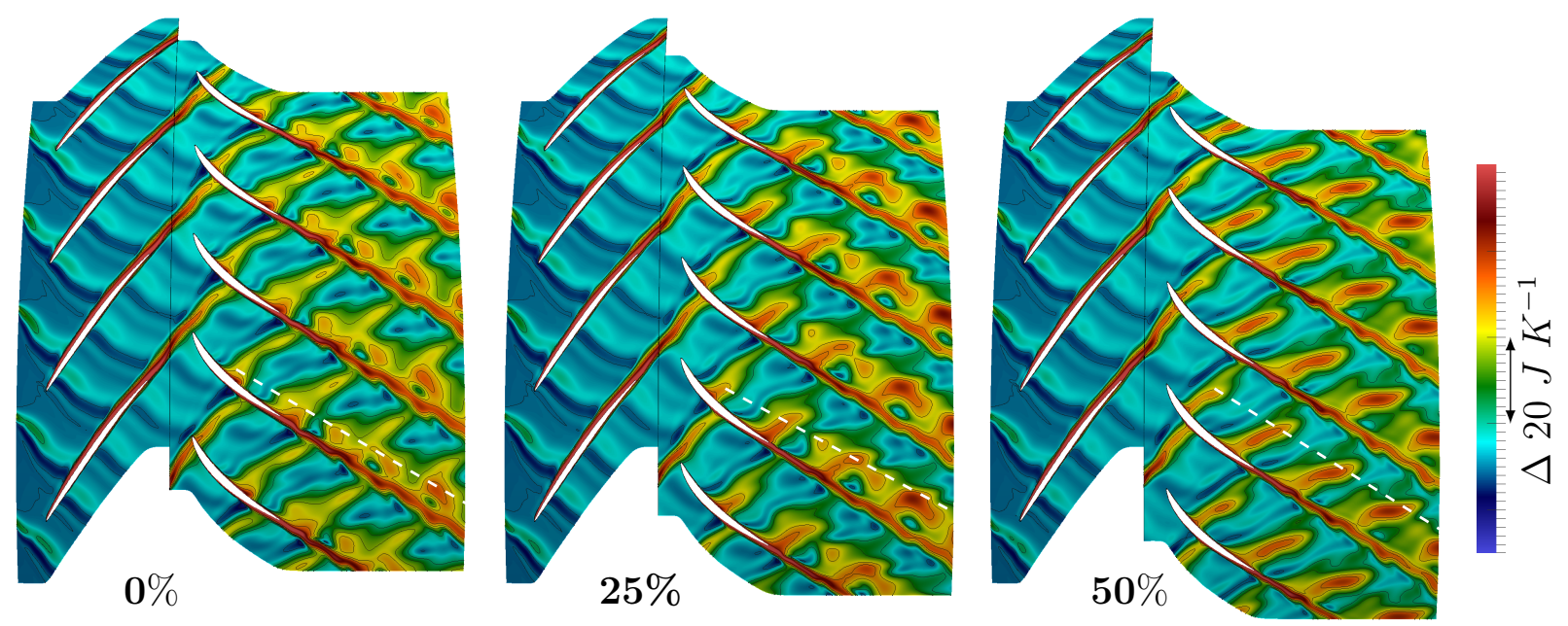

Figure 3. The entropy contours on the $95 \%$ span illustrate the instantaneous wake pattern for the different stator clocking configurations. (Differences can be only observed in the downstream stator vanes, due to the convective nature of entropy.) A white dashed line indicates the wake induced by the upstream stator. 

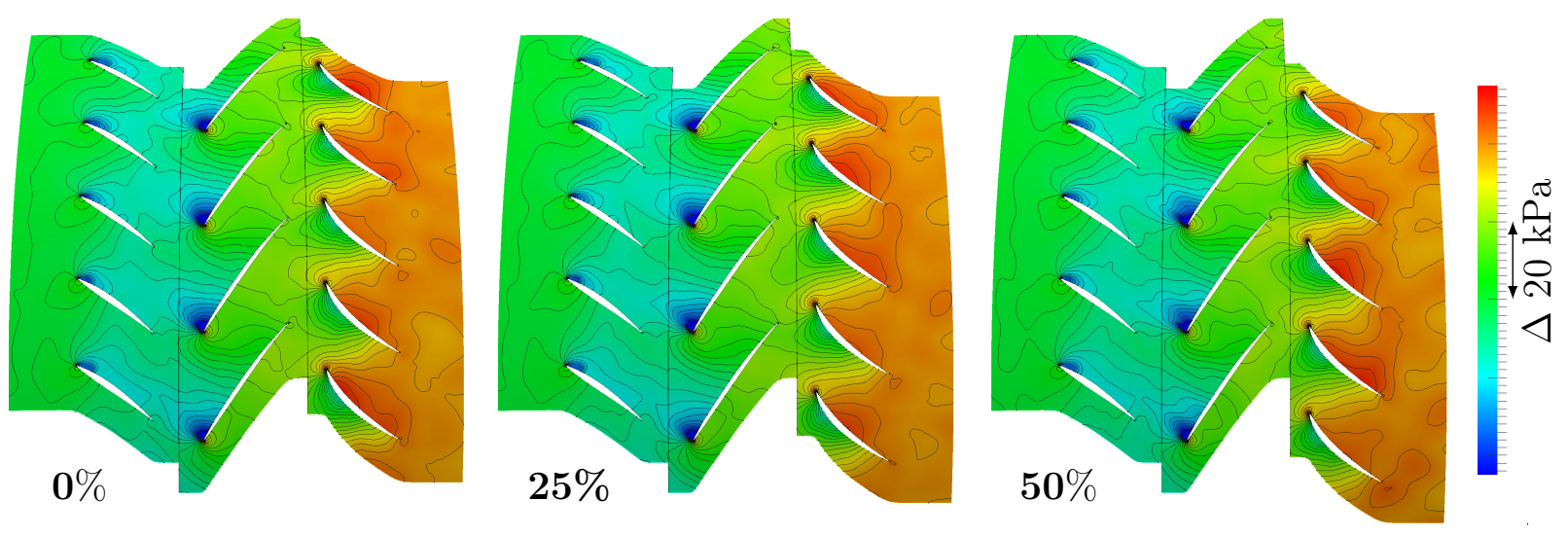

Figure 4. The static pressure contours on the $95 \%$ span show the instantaneous potential field interaction for the different stator clocking configurations.

field. With the rotor interaction, the acoustic wave generation and reflection behaviour is influenced and passed on to the VIGV's. Figure 4 indicates that the hydrodynamic pressure distributions at the VIGV are only moderately altered.

\section{III.A. Acoustic noise}

For acoustic noise considerations, the pressure waves propagating upstream are of interest, which exhibit smaller amplitudes than the hydrodynamic pressure fluctuations discussed in the prior section. The sound pressure levels radiated upstream, in terms of the Tyler and Sofrin ${ }^{5}$ modes, are illustrated in Fig. 5 for the blade passing frequency and its first two harmonics. Only a few circumferential modes (being cut-on) govern the far field tonalities exhibiting at least $10 \mathrm{~dB}$ higher magnitudes as the cut-off modes.

Comparing the relative mode amplitudes between clocking configurations shown in Fig. 5, minor variations $(<2 d B)$ can be observed for the cut-on modes at the blade passing frequency and its first harmonic. However, for the second harmonic, stator clocking has higher impact (up to $10 \mathrm{~dB}$ ) on the cut-on mode amplitudes. Also for cut-off (higher interaction order) modes, the respective stator location is significant.
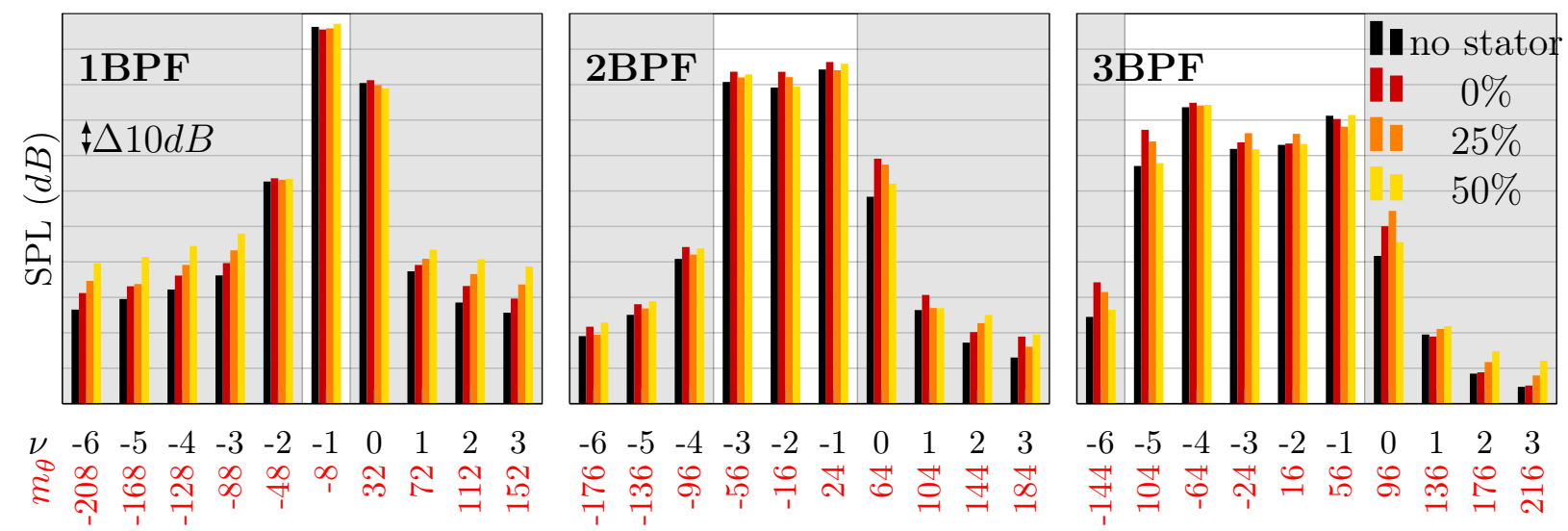

Figure 5. Calculated sound pressure levels of the circumferential modes for the different stator clocking configurations. The cut-off criteria is indicated by the grey shaded areas. 

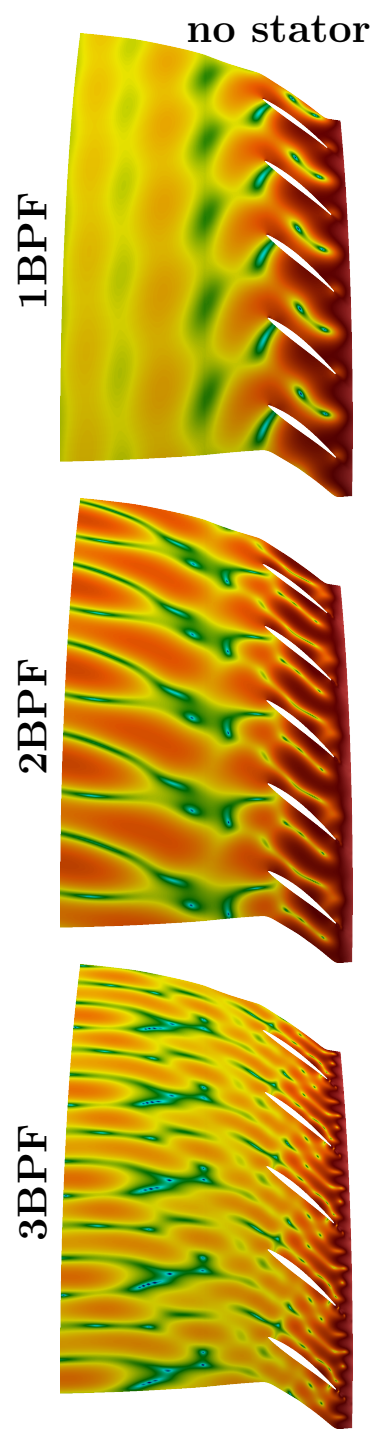
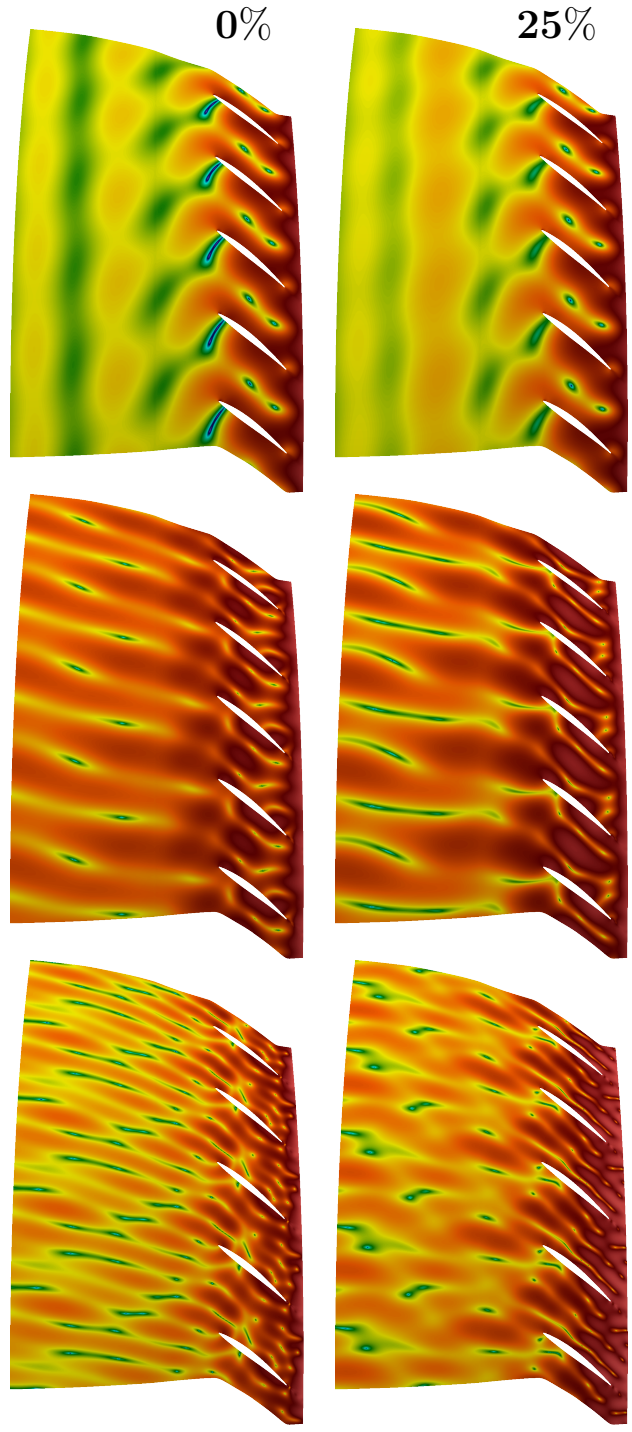
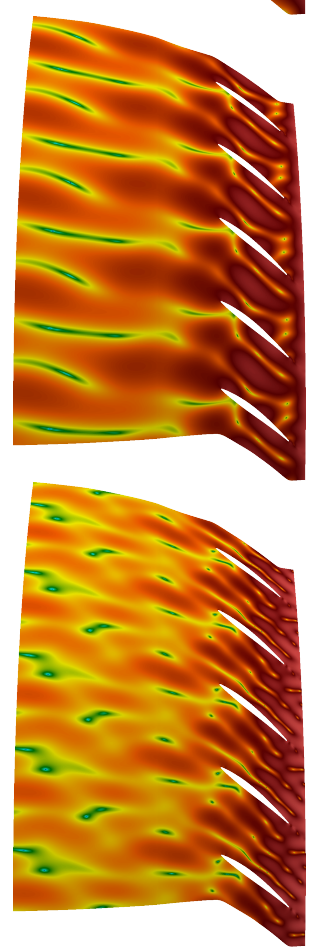
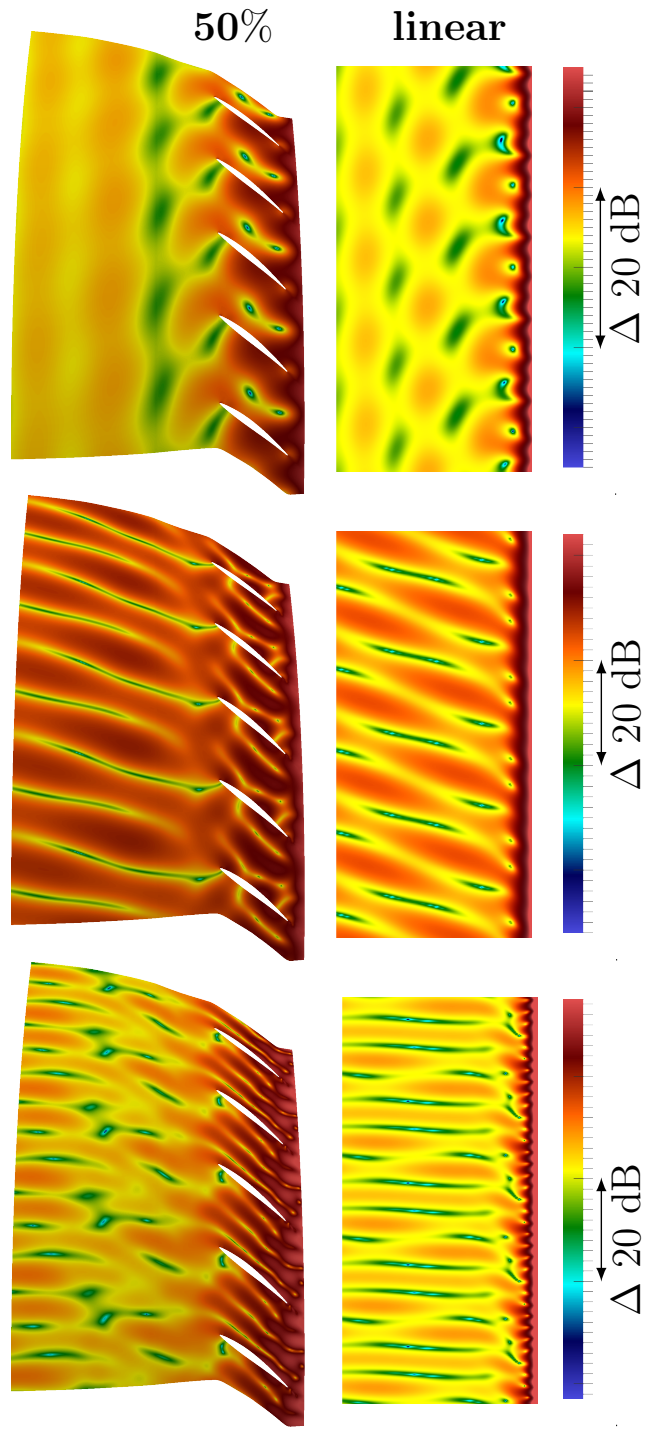

Figure 6. For the blade passing frequency and the first two harmonics, the sound pressure level contours are shown on the VIGV casing (for location orientation see Fig. 2).

Upstream of the VIGV's, the tonal sound pressure contours reveal the interference pattern provoked by the acoustic modes, which are show in Fig. 6 and Fig. 7, respectively for selected clocking configurations. The complex interplay of pressure waves exhibits regions where the amplitudes fade, which is a clear indication of wave interference. (Rather large blurred areas are visible for the configuration without downstream stator at 2 and 3 times the blade passing frequency in Fig. 7.) These interference patterns cause elaborate sound pressure level contours, which should be considered for optimal design of acoustic liners in the near field. Locations, where high sound pressure levels occur, are preferred for the application of Helmholtz resonators, because their efficiency is better with higher pressure fluctuations. In contrast, areas with low sound pressure levels are favourably used for other frequencies. Thus, plotting an overlay can give a simple indicator for which frequency the Helmholtz resonators shall be designed in a certain area.

Clear trends of sound pressure contours can be observed comparing the clocked configurations. The interference amplifies, decays, or shifts to another pattern, which indicates 

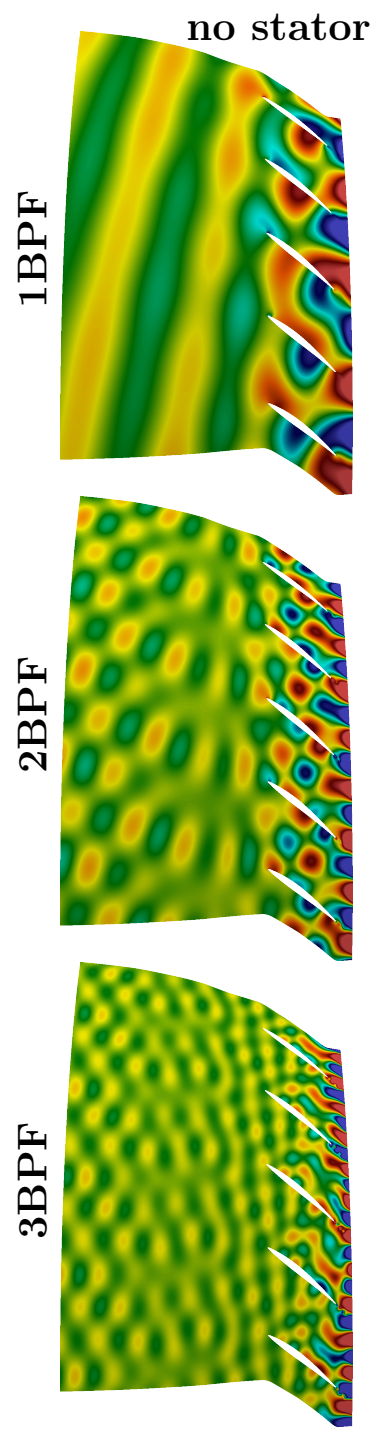
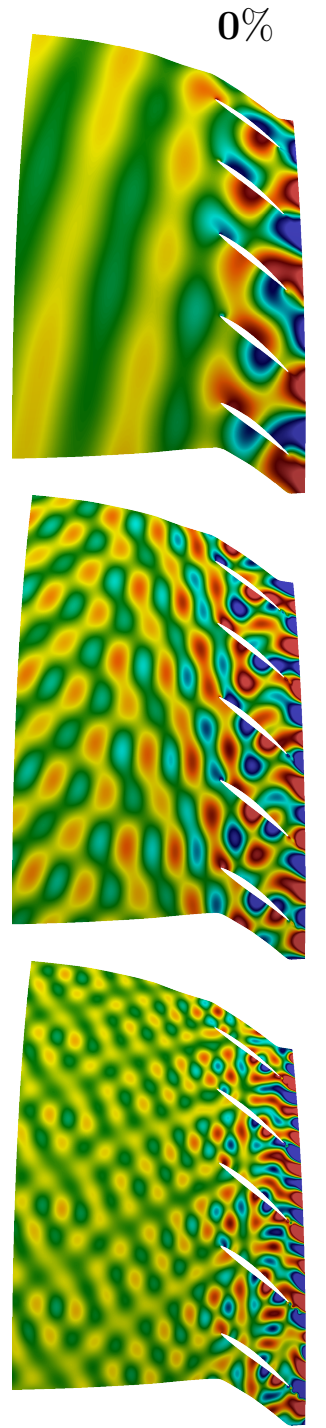
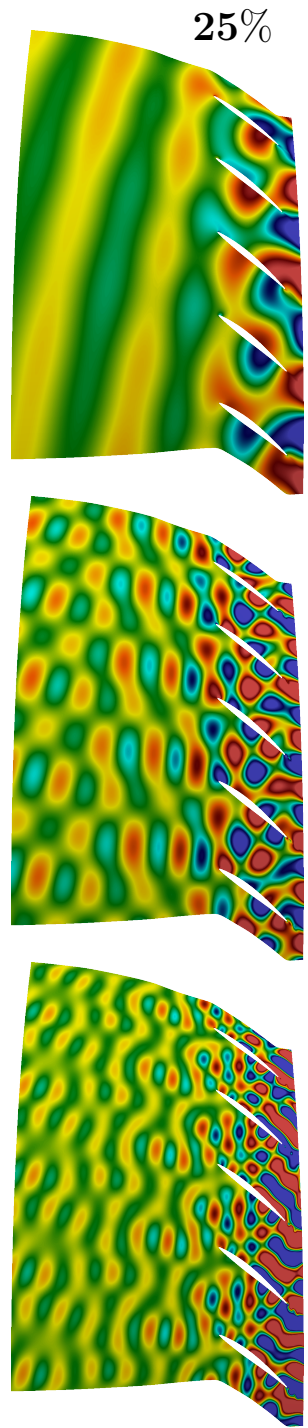
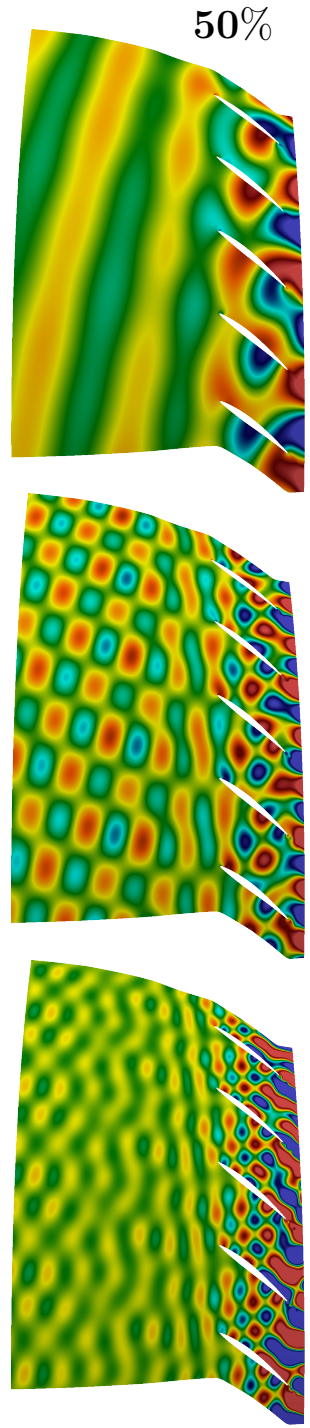

are shown on the VIGV casing.

that the phase of certain modes is altered with stator clocking. Note that Fig. 5 showed minor changes in the mode amplitudes for different cases. The configuration without downstream stator provokes sound pressure level contours with similarities to the $50 \%$ clocking configuration.

\section{III.B. Low-order modelling}

Although having the full data available of the high fidelity simulations, it remains challenging to interpret the flow field. Low-order methods allow additional investigation of the acoustic field by analysing the individual circumferential modes, while their superposition represents the full sound pressure levels. Figure 7 shows that low-order models can predict accurately the inclination angle of the pressure waves and cut-off behaviour corresponding to particular modes. Their combination leads to a reasonable estimation of the sound pressure levels upstream of the VIGV's, as shown in Fig. 6. (The amplitudes of the modes are extracted from 


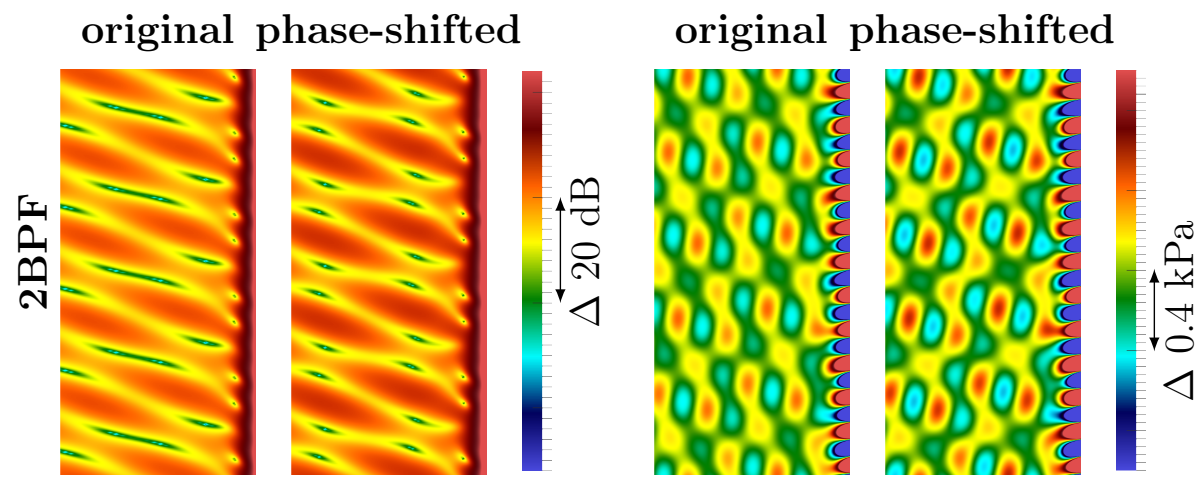

Figure 8. Effect of phase lag adjustment in the low-order model for selected modes on the sound pressure level contours (left) and the instantaneous pressure fluctuations (right).

the high fidelity simulations, where a phase shift has been neglected.) It may be highlighted that a complete match is not expected, because the sound pressure level contours are plotted on the VIGV casing and at constant radius for the high fidelity and low-order calculations, respectively. Further, the low-order model does not consider a respective phase shift of the modes. By adjusting the phase component of particular modes, the sound pressure level contours can be matched better to particular stator clocking configurations.

At least four modes were required to reasonably reconstruct the sound pressure levels for the blade passing frequency at the VIGV casing. Solely one mode, i.e. $m_{\theta}=-8$, is cut-on. (Note that a single acoustic wave would cause a smooth sound pressure level distribution on the VIGV casing.) The modes, $m_{\theta}=-48$ and $m_{\theta}=32$, are cut-off, but contribute significantly in the near field. Their presence is indicated in Fig. 6 by the inclined sound pressure level amplification pattern dying out slightly upstream of the S-duct. Interesting to observe is the vertical sound pressure level interference pattern becoming only apparent for the blade passing frequency. This can only be generated via the interaction of downstream travelling waves. With this indication, the occurrence of reflections has been detected at the bottom wall of the S-duct in the high fidelity simulations. This reflected wave has been modelled with an elongated wavelength (accordingly to the smooth bend of the S-duct) and an eight times reduced amplitude with respect to the radiated mode amplitude.

To predict the sound pressure level contours for the higher harmonics illustrated in Fig. 6, all modes displayed in Fig. 5 have been conjoined. The computation using the low-order model took few milliseconds. Although, the inter-phase lag between modes can be adjusted to obtain better comparison to particular cases (as shown in Fig. 8), the effect of duct curvature is evidently distorting the wave propagation.

\section{Conclusions}

The influence of stator clocking on the acoustic signature of a typical one-and-a-half stage axial high-pressure compressor has been investigated. Employing high fidelity numerical simulations, the acoustic noise source generation mechanisms of rotor/stator interaction have been analysed.

Stator clocking was observed to have negligible impact of the compressor efficiency $(<$ $0.02 \%$ and is in accordance with literature) and tonal acoustic noise levels, in terms of cut- 
on modes, received in the far field. The $10 d B$ higher amplitudes of the cut-on Tyler and Sofrin modes suggested dominance over the cut-off modes. In the near field, the Fourier filtered wave pattern at the blade passing frequency and its first two harmonics showed that the amplitudes evolved different when the clocking position is varied and that cutoff modes influence the acoustic pressure fluctuations. The sound pressure level contours (manifested as elaborate interference pattern on the compressor casing) exhibited significant transformations and the nodal location changed. Such sound pressure contour maps can be used for optimal design of acoustic liners in the near field of the blades, where Helmholtz resonators should be positioned at high sound pressure levels to maximise their efficiency.

A simplistic low-order model was used to estimate the wave propagation direction of particular acoustic modes. The comparison between high fidelity and low-order simulation results revealed that this can be achieved with satisfactory agreement, which further represents a verification of the high fidelity simulation results. Further, the cut-off criteria for the circumferential modes was predicted in accordance with the high fidelity results. It was shown that cut-off modes need to be considered to predict the sound pressure levels in the near field, because these contribute significantly to the shape of the interference pattern. Hence, cut-off modes need to be considered designing acoustic liners close to blade rows.

Applying the mode amplitudes from the high fidelity calculations in the low-order model, the interference pattern of the sound pressure levels in the near field were accurately estimated, although duct curvature was not considered. Together with its computational efficiency, the low-order model revealed promising capabilities as future design tool. Nonetheless, the absence of phase information in the low-order formulation challenges the predictive capacities with many modes interacting. It has been shown that the match with simulation results can be improved by adapting this relative phase shift between modes. These observed limitations of the low-order model suggest that further development is needed.

\section{Acknowledgments}

The authors wish to express their sincere gratitude to Rolls-Royce plc for the permission to publish this paper, which partly developed through the Rolls-Royce plc and Innovate UK Aerospace Technology Institute funded research programme, ACAPELLA.

\section{References}

${ }^{1}$ Griffiths, J., "The spectrum of compressor noise of a jet engine," Journal of Sound and Vibration, Vol. 1, No. 2, 1964, pp. 127129-128140.

${ }^{2}$ Landström, U., Kjellberg, A., Söderberg, L., and Nordström, B., "The effects of broadband, tonal and masked ventilation noise on performance, wakefulness and annoyance," Journal of Low Frequency Noise, Vibration and Active Control, Vol. 10, No. 4, 1991, pp. 112-122.

${ }^{3}$ Lowson, M., "Reduction of compressor noise radiation," The Journal of the Acoustical Society of America, Vol. 43, No. 1, 1968, pp. 37-50.

${ }^{4}$ Kemp, N. H. and Sears, W. R., "Aerodynamic interference between moving blade rows," Journal of the Aeronautical Sciences, Vol. 20, 1953, pp. 585-598.

${ }^{5}$ Tyler, J. M. and Sofrin, T. G., "Axial flow compressor noise studies," Tech. rep., SAE Technical Paper, 1962.

${ }^{6}$ Copeland, W. L., Crigler, J. L., and Dibble Jr, A. C., "Contribution of downstream stator to the interaction noise of a single-stage axial-flow compressor," NASA Technical Note TN D-3892, 1967. 
${ }^{7}$ Walker, G. J. and Oliver, A., "The effect of interaction between wakes from blade rows in an axial flow compressor on the noise generated by blade interaction," Journal of Engineering for Power, Vol. 94, No. 4, 1972, pp. 241-248.

${ }^{8}$ Schmidt, D. P. and Okiishi, T. H., "Multistage axial-flow turbomachine wake production, transport, and interaction," AIAA Journal, Vol. 15, No. 8, 1977, pp. 1138-1145.

${ }^{9}$ Kovalev, D., Saren, V., and Shipov, R., "Influence of Mutual Circumferential Shift of Stators on the Noise Generated by System of Rows Stator -Rotor-Stator of the Axial Compressor," Unsteady Aerodynamics, Aeroacoustics and Aeroelasticity of Turbomachines, Springer, 2006, pp. 261-273.

${ }^{10}$ Kamiyoshi, S. and Kaji, S., "Application of airfoil clocking technology to reduction of multi-stage fan tone," rth AIAA/CEAS Aeroacoustics Conference and Exhibit, AIAA Paper No. 2001-2149, May 2001.

${ }^{11}$ Kamiyoshi, S. and Kaji, S., "Tone noise reduction of multi-stage fan by airfoil clocking," 6th Aeroacoustics Conference and Exhibit, AIAA Paper No. 2000-1992, 2000.

${ }^{12}$ Barankiewicz, W. S. and Hathaway, M. D., "Effects of Stator Indexing on Performance in a Low Speed Multistage Axial Compressor," ASME 1997 International Gas Turbine and Aeroengine Congress and Exhibition, American Society of Mechanical Engineers, 1997, pp. V001T03A098-V001T03A098.

${ }^{13}$ Saren, V., Savin, N., Dorney, D., and Zacharias, R., "Experimental and numerical investigation of unsteady rotor-stator interaction on axial compressor stage (with IGV) performance," Unsteady Aerodynamics and Aeroelasticity of Turbomachines, Springer, 1998, pp. 407-424.

${ }^{14}$ Salontay, J., Key, N., and Fulayter, R., "A Computational Investigation of Vane Clocking Effects on Embedded Compressor Stage Efficiency," 46th AIAA/ASME/SAE/ASEE Joint Propulsion Conference \& Exhibit, 2010, p. 6534.

${ }^{15}$ Städing, J., Friedrichs, J., Waitz, T., Dobriloff, C., Becker, B., and Gümmer, V., "The Potential of Rotor and Stator Clocking in a 2.5-Stage Low-Speed Axial Compressor," ASME Turbo Expo 2012: Turbine Technical Conference and Exposition, American Society of Mechanical Engineers, 2012, pp. 2453-2466.

${ }^{16}$ Huang, H., Yang, H., Feng, G., and Wang, Z., "Clocking effect in a two-stage compressor with different inter-blade-row gaps," Journal of Thermal Science, Vol. 13, No. 1, 2004, pp. 8-15.

${ }^{17}$ Dorney, D. J., Sharma, O. P., and Gundy-Burlet, K. L., "Physics Of Airfoil Clocking In A High-Speed Axial Compressor," International Journal of Turbo and Jet Engines, Vol. 15, No. 4, 1998, pp. 259-270.

${ }^{18}$ Key, N. L., Lawless, P. B., and Fleeter, S., "An experimental study of vane clocking effects on embedded compressor stage performance," Journal of turbomachinery, Vol. 132, No. 1, 2010, pp. 011018.

${ }^{19}$ Gundy-Burlet, K. L., Dorney, D. J., and St Whitney, P., "Physics of Airfoil Clocking in Axial Compressors," ASME 1997 International Gas Turbine and Aeroengine Congress and Exhibition, American Society of Mechanical Engineers, 1997, pp. V004T14A058-V004T14A058.

${ }^{20}$ He, L., Chen, T., Wells, R., Li, Y., and Ning, W., "Analysis of rotor-rotor and stator-stator interferences in multi-stage turbomachines," Transactions of the American Society of Mechanical Engineers Journal of Turbomachinery, Vol. 124, No. 4, 2002, pp. 564-571.

${ }^{21}$ Fruth, F., Vogt, D. M., Bladh, R., and Fransson, T. H., "Unsteady Forcing vs. Efficiency: The Effect of Clocking on a Transonic Industrial Compressor," ASME 2013 Fluids Engineering Division Summer Meeting, American Society of Mechanical Engineers, 2013, pp. V01AT02A010-V01AT02A010.

${ }^{22}$ Lapworth, L., "Hydra-CFD: a framework for collaborative CFD development," International Conference on Scientific and Engineering Computation (IC-SEC), Singapore, June, Vol. 30, 2004.

${ }^{23}$ Annex 16 - Environmental Protection, "Procedures for the Noise Certification of Aircraft," International Civil Aviation Organisation, 1981.

${ }^{24}$ Milli, A. and Shahpar, S., "PADRAM: Parametric Design and Rapid Meshing System for Complex Turbomachinery Configurations," ASME Turbo Expo 2012: Turbine Technical Conference and Exposition, American Society of Mechanical Engineers, 2012, pp. 2135-2148.

${ }^{25}$ Law, T. R., Corral, R., Fernández, J. R., and Serrano, A., "Linear viscous eigenmode analysis within a radially varying swirling flow," AIAA paper, , No. 2010-3890, 2010.

${ }^{26}$ Courtiade, N., Ottavy, X., and Gourdain, N., "Modal decomposition for the analysis of the rotor-stator interactions in multistage compressors," Journal of Thermal Science, Vol. 21, No. 3, 2012, pp. 276-285. 